Research Article

\title{
On Iterative Learning Control for Remote Control Systems with Packet Losses
}

\author{
Chunping Liu, ${ }^{1,2}$ Rong Xiong, Jianxin $\mathrm{Xu},{ }^{1,3}$ and Jun $\mathrm{Wu}^{1}$ \\ ${ }^{1}$ State Key Laboratory of Industrial Control Technology, Institute of Cyber-Systems and Control, \\ Zhejiang University, Hangzhou 310027, China \\ ${ }^{2}$ Department of Electric and Information Engineering, Shaoxing College of Arts and Sciences, Shaoxing 31200, China \\ ${ }^{3}$ Department of Electrical and Computer Engineering, National University of Singapore, Singapore 117576
}

Correspondence should be addressed to Rong Xiong; rxiong@iipc.zju.edu.cn

Received 16 May 2013; Accepted 8 October 2013

Academic Editor: Neal N. Xiong

Copyright (C) 2013 Chunping Liu et al. This is an open access article distributed under the Creative Commons Attribution License, which permits unrestricted use, distribution, and reproduction in any medium, provided the original work is properly cited.

The problem of iterative learning control (ILC) is considered for a class of time-varying systems with random packet dropouts. It is assumed that an ILC scheme is implemented via a remote control system and that packet dropout occurs during the packet transmission between the ILC controller and the actuator of remote plant. The packet dropout is viewed as a binary switching sequence which is subject to the Bernoulli distribution. In order to eliminate the effect of packet dropouts on the convergence property of output error, the hold-input scheme is adopted to compensate the packet dropout at the actuator. It is shown that the hold-input scheme with average ILC can achieve asymptotical convergence along the iteration axis for discrete time-varying linear system. Numerical examples are provided to show the effectiveness of the proposed method.

\section{Introduction}

Iterative learning control (ILC) is an attractive technique when dealing with systems that execute the same task repeatedly over a finite time interval [1]. This technique has been the center of interest of many researchers over the two decades [2-5] and covered a wide scope of research issues such as model uncertainty [6-8], disturbance uncertainty and stochastic noise [9], the initial condition and desired trajectory uncertainty [10-12], continuous-time nonlinear system control [13], and parameter interval uncertainty [14].

On the other hand, the remote control systems have been the focus of several research studies over the last few years [15-21]. In the remote control systems, one feature is that the control loops are closed through a real-time communication channel which transmits signals from the sensors to the controller and from the controller to the actuators [17]. The remote control systems eliminate unnecessary wiring reducing the complexity and overall cost in designing and implementing the control systems. However, the introduction of communication networks makes the analysis and control design more complicated than classical feedback loops. Data packet dropout can randomly occur due to node failure or network congestion and impose one of the most important issues in remote control systems. In $[18,19]$, the authors are concerned with the stability problem for remote control systems with the packet dropout. In the work [20, 21], decentralized stabilization of remote control systems with nonlinear perturbations is studied.

Besides the stability issue, trajectory tracking is a challenging issue for remote control systems. Fortunately, for periodic systems, iterative learning control offers a systematic design that can improve the tracking performance by iterations in a fixed time interval. ILC is in principle a feedforward technique; thus it can send the controller signals obtained from previous trials. It is still an open research area in ILC which is implemented via a remote systems setting, except for certain pioneer works [22-29]. In [22, 23], the authors designed an optimal ILC controller for a class of linear systems with random packet dropouts. Bu et al. [26] studied the stability of first and high order ILC with data dropout when the plant is subject to measurement signal dropout. In $[24,25]$, the authors investigated the implementation of ILC in a remote control systems environment and specifically 
focused on compensation when both random data dropouts and delays occur at the communication network between the sensors and the controller. In [27], a sampled-data ILC approach was proposed for a class of nonlinear remote control systems to analyze the effect of packet loss. In [28], the author considered the problem of ILC for a class of nonlinear systems with control signal dropouts and measurement signal dropouts, but the convergence analysis needs controller and actuator to know the received signal whether lost or not. Huang and Fang [29] discussed the wireless remote iterative learning control system with random data dropouts.

In this paper, we proposed an ILC for a time-varying system with random packet dropouts. As depicted in previous studies [22-29], there are two different kinds of packet dropouts in remote ILC systems: control input signal dropouts and output measurement signal dropouts. For the sake of convenience, we only consider the control signal dropouts in this paper, but the results can be extended to the measurement signal dropouts. The packet dropouts would be described as a binary sequence which is subject to a Bernoulli distribution taking the value of one or zero with certain probability. The ILC law adopts an iteration-average operator and a revised learning gain that takes into consideration the probabilities of data-dropout factors. As a result, the ensemble average of the output tracking errors can be made to converge along the iteration axis. In this paper, we consider a class of discrete time linear plants with output matrix $\mathbf{C}$ and input matrix B; our results refer only to $\mathbf{C B}$ of full-column rank.

The paper is organized as follows. Section 2 formulates the system problem. Section 3 formulates the hold-input scheme with average ILC algorithm and proves the convergence property of ILC for linear varying discrete-time plants. Section 4 presents numerical examples, and Section 5 draws the conclusions.

\section{Problem Formulation}

Consider the ILC system with network communication depicted in Figure 1. The discrete time linear plant with actuators and sensors is described by

$$
\begin{aligned}
& \mathbf{x}_{i}(t+1)=\mathbf{A}(t) \mathbf{x}_{i}(t)+\mathbf{B}\left(\mathbf{u}_{i}(t)+\mathbf{w}_{i}(t)\right) \\
& \mathbf{y}_{i}(t)=\mathbf{C} \mathbf{x}_{i}(t)+\mathbf{v}_{i}(t),
\end{aligned} \quad t \in\{0,1,2, \ldots, T\},
$$

where $i \in \mathbb{Z}_{+}$denotes the iteration index; $T \in \mathbb{Z}_{+}$is a given finite time; $\mathbf{x}_{i}(t) \in \mathbb{R}^{n}, \mathbf{u}_{i}(t) \in \mathbb{R}^{q}$, and $\mathbf{y}_{i}(t) \in \mathbb{R}^{m}$ are state, control, and output, respectively; $\mathbf{A}(t) \in \mathbb{R}^{n \times n}$ is unknown matrix, while $\mathbf{B} \in \mathbb{R}^{n \times q}$ and $\mathbf{C} \in \mathbb{R}^{m \times n}$ are known; $\mathbf{w}_{i}(t) \in \mathbb{R}^{q}$ and $\mathbf{v}_{i}(t) \in \mathbb{R}^{m}$ are random noises with $\mathscr{E}\left[\mathbf{w}_{i}(t)\right]=\mathbf{0}$ and $\mathscr{E}\left[\mathbf{v}_{i}(t)\right]=\mathbf{0}$; for all $i \in \mathbb{Z}_{+}$, the initial state $\mathbf{x}_{i}(0)$ is a random variable of $\mathscr{E}\left[\mathbf{x}_{i}(0)\right]=\mathbf{x}_{0}$ with a fixed point $\mathbf{x}_{0} \in \mathbb{R}^{n}$. Assume that $\mathbf{C B}$ has full-column rank. The discrete time controller consists of a ILC algorithm and a memory. The controller and the actuators are connected via a communication network through which the controller transmits data to the actuators, while the controller is directly connected to the sensors. The plant and the controller are assumed to be time driven and synchronized.

At each $t \in\{0, \ldots, T\}$ of the $i$ th iteration stage, the controller output $\widehat{\mathbf{u}}_{i}(t)$ is computed, the controller transmits $\widehat{\mathbf{u}}_{i}(t)$ to the actuators through the network. The transmission may succeed or fail. For a successful transmission, it is assumed that the transmission delay through the network is negligible. With the negligible delay, the actuators can employ $\mathbf{u}_{i}(t)=$ $\widehat{\mathbf{u}}_{i}(t)$, when $\widehat{\mathbf{u}}_{i}(t)$ is transmitted successfully. Of course, when the transmission fails, the actuators receive no $\widehat{\mathbf{u}}_{i}(t)$ and have to employ $\mathbf{u}_{i}(t)=\mathbf{u}_{i}(t-1)$ (this paper prescribes $\mathbf{u}_{i}(-1)=0$ ). Overall, the scheme of actuators is

$$
\mathbf{u}_{i}(t)=\gamma_{i}(t) \widehat{\mathbf{u}}_{i}(t)+\left(1-\gamma_{i}(t)\right) \mathbf{u}_{i}(t-1),
$$

where

$$
\gamma_{i}(t)= \begin{cases}1, & \text { if the transmission of } \widehat{\mathbf{u}}_{i}(t) \text { succeeds } \\ 0, & \text { if the transmission of } \widehat{\mathbf{u}}_{i}(t) \text { fails. }\end{cases}
$$

Specially, this paper assumes that, for all $i \in \mathbb{Z}_{+}$, for all $t \in\{0, \ldots, T\}, \gamma_{i}(t)$ is a random variable of $\mathscr{E}\left[\gamma_{i}(t)\right]=$ $\gamma$ with a constant $\gamma \in(0,1)$ as well as that $\gamma_{i}\left(t_{1}\right)$ and $\gamma_{j}\left(t_{2}\right)$ are independent either when $i \neq j$ or when $t_{1} \neq t_{2}$. In addition, TCP-like protocol is assumed, in which there is an acknowledgment for a successful transmission, and hence the controller has indicators of whether the current controller output is received or not by the actuators.

Assumption 1. Given an output reference trajectory $\mathbf{y}_{d}(t)$, which is realizable; that is, there exists a unique desired control input $\mathbf{u}_{d}(t) \in \mathbb{R}^{q}$ such that

$$
\begin{aligned}
& \mathbf{x}_{d}(t+1)=\mathbf{A}(t) \mathbf{x}_{d}(t)+\mathbf{B} \mathbf{u}_{d}(t) \\
& \mathbf{y}_{d}(t)=\mathbf{C} \mathbf{x}_{d}(t),
\end{aligned}
$$

The purpose of this paper is to design an iterative learning control law for the above plant with network communication such that $\mathbf{y}_{i}(t)$ tracks $\mathbf{y}_{d}(t)$ as closely as possible when $i$ is large enough.

\section{ILC Algorithms and Convergence Analysis}

Denote $\mathbf{e}_{i}(t) \triangleq \mathbf{y}_{d}(t)-\mathbf{y}_{i}(t)$. The control law is a D-type ILC with average operator that employs updating mechanism:

$$
\begin{aligned}
\widehat{\mathbf{u}}_{i+1}(t) & =\frac{1}{i} \sum_{j=1}^{i} \mathbf{u}_{j}(t)+\frac{i+1}{i} \mathbf{L} \sum_{j=1}^{i} \mathbf{e}_{j}(t+1) \\
& =\mathscr{A}\left[\mathbf{u}_{i}(t)\right]+(i+1) \mathbf{L} \mathscr{A}\left[\mathbf{e}_{i}(t+1)\right],
\end{aligned}
$$

where the gain matrix $\mathbf{L} \in \mathbb{R}^{q \times m}$. From (2) and (5), the holdinput scheme with average ILC is expressed as

$$
\begin{aligned}
\mathbf{u}_{i+1}(t)= & \gamma_{i+1}(t) \widehat{\mathbf{u}}_{i+1}(t)+\left(1-\gamma_{i+1}(t)\right) \mathbf{u}_{i+1}(t-1) \\
= & \gamma_{i+1}(t)\left(\mathscr{A}\left[\mathbf{u}_{i}(t)\right]+(i+1) \mathbf{L} \mathscr{A}\left[\mathbf{e}_{i}(t+1)\right]\right) \\
& +\left(1-\gamma_{i+1}(t)\right) \mathbf{u}_{i+1}(t-1) .
\end{aligned}
$$




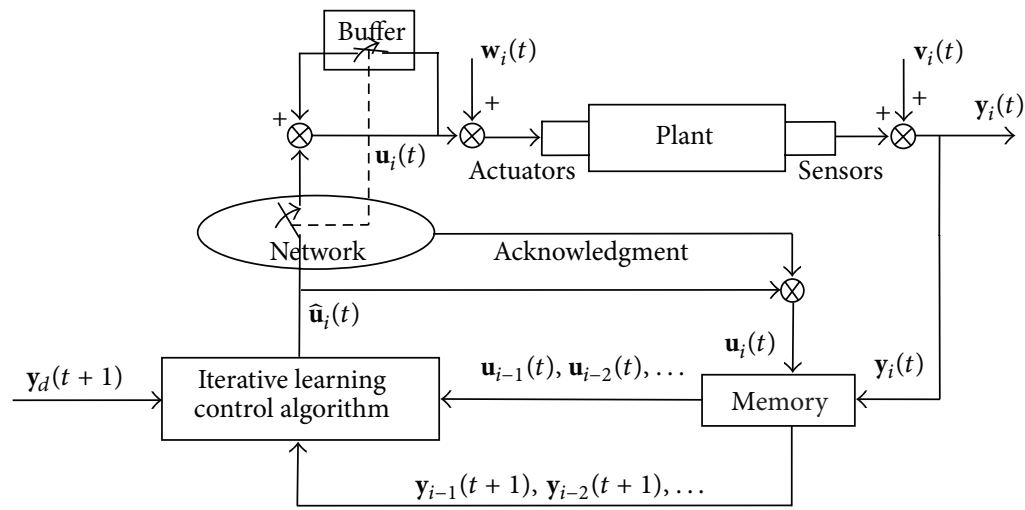

FIGURE 1: The schematic diagram of the networked control system.

Define the input and state errors

$$
\begin{aligned}
& \Delta \mathbf{u}_{i+1}(t) \triangleq \mathbf{u}_{d}(t)-\mathbf{u}_{i+1}(t), \\
& \Delta \mathbf{x}_{i+1}(t) \triangleq \mathbf{x}_{d}(t)-\mathbf{x}_{i+1}(t) .
\end{aligned}
$$

And subtracting $\mathbf{u}_{d}(t)$ from both sides of (6) yields

$$
\begin{aligned}
\Delta \mathbf{u}_{i+1}(t)= & \gamma_{i+1}(t)\left(\mathscr{A}\left[\Delta \mathbf{u}_{i}(t)\right]-(i+1) \mathbf{L} \mathscr{A}\left[\mathbf{e}_{i}(t+1)\right]\right) \\
& +\left(1-\gamma_{i+1}(t)\right)\left(\Delta \mathbf{u}_{i+1}(t-1)+\boldsymbol{\delta}(t)\right)
\end{aligned}
$$

where (this paper prescribes $\mathbf{u}_{d}(-1)=0$ and hence $\boldsymbol{\delta}(0)=$ $\left.\mathbf{u}_{d}(0)\right) \boldsymbol{\delta}(t) \triangleq \mathbf{u}_{d}(t)-\mathbf{u}_{d}(t-1)$. Noticing that $\gamma_{i+1}(t)$ is independent of $\mathscr{A}\left[\Delta \mathbf{u}_{i}(t)\right], \mathscr{A}\left[\mathbf{e}_{i}(t+1)\right]$ and $\Delta \mathbf{u}_{i+1}(t-1)$ and taking expectation on both sides of (8), we have

$$
\begin{aligned}
\mathscr{E}\left[\Delta \mathbf{u}_{i+1}(t)\right] \\
=\gamma\left(\mathscr{E}\left[\mathscr{A}\left[\Delta \mathbf{u}_{i}(t)\right]\right]-(i+1) \mathbf{L} \mathscr{E}\left[\mathscr{A}\left[\mathbf{e}_{i}(t+1)\right]\right]\right) \\
\quad+(1-\gamma)\left(\mathscr{E}\left[\Delta \mathbf{u}_{i+1}(t-1)\right]+\boldsymbol{\delta}(t)\right) .
\end{aligned}
$$

Expanding expression (9) from $\mathscr{E}\left[\Delta \mathbf{u}_{i+1}(t-1)\right]$ to $\mathscr{E}\left[\Delta \mathbf{u}_{i+1}(0)\right]$, we have

$$
\begin{aligned}
\mathscr{E}\left[\Delta \mathbf{u}_{i+1}(t-1)\right] & \\
= & \gamma\left(\mathscr{E}\left[\mathscr{A}\left[\Delta \mathbf{u}_{i}(t-1)\right]\right]-(i+1) \mathbf{L} \mathscr{E}\left[\mathscr{A}\left[\mathbf{e}_{i}(t)\right]\right]\right) \\
& +(1-\gamma)\left(\mathscr{E}\left[\Delta \mathbf{u}_{i+1}(t-2)\right]+\boldsymbol{\delta}(t-1)\right),
\end{aligned}
$$

$$
\begin{aligned}
\mathscr{E}\left[\Delta \mathbf{u}_{i+1}(t-2)\right] & \\
= & \gamma\left(\mathscr{E}\left[\mathscr{A}\left[\Delta \mathbf{u}_{i}(t-2)\right]\right]-(i+1) \mathbf{L} \mathscr{E}\left[\mathscr{A}\left[\mathbf{e}_{i}(t-1)\right]\right]\right) \\
& +(1-\gamma)\left(\mathscr{E}\left[\Delta \mathbf{u}_{i+1}(t-3)\right]+\boldsymbol{\delta}(t-2)\right) \\
& \vdots \\
\mathscr{E}\left[\Delta \mathbf{u}_{i+1}(0)\right] & \\
= & \gamma\left(\mathscr{E}\left[\mathscr{A}\left[\Delta \mathbf{u}_{i}(0)\right]\right]-(i+1) \mathbf{L} \mathscr{E}\left[\mathscr{A}\left[\mathbf{e}_{i}(1)\right]\right]\right) \\
& +(1-\gamma)\left(\mathscr{E}\left[\Delta \mathbf{u}_{i+1}(-1)\right]+\boldsymbol{\delta}(0)\right) \\
= & \gamma\left(\mathscr{E}\left[\mathscr{A}\left[\Delta \mathbf{u}_{i}(0)\right]\right]-(i+1) \mathbf{L} \mathscr{E}\left[\mathscr{A}\left[\mathbf{e}_{i}(1)\right]\right]\right) \\
& +(1-\gamma) \boldsymbol{\delta}(0) .
\end{aligned}
$$

The above expression can be arranged later below (this paper prescribes $\sum_{k=k_{1}}^{k_{2}}=0$ when $k_{2}<k_{1}$ )

$$
\begin{aligned}
\mathscr{E}\left[\Delta \mathbf{u}_{i+1}(t-1)\right]= & \sum_{k=0}^{t-1} \gamma(1-\gamma)^{k} \mathscr{E}\left[\mathscr{A}\left[\Delta \mathbf{u}_{i}(t-k-1)\right]\right] \\
& -(i+1) \mathbf{L} \sum_{k=0}^{t-1} \gamma(1-\gamma)^{k} \mathscr{E}\left[\mathscr{A}\left[\mathbf{e}_{i}(t-k)\right]\right] \\
& +\sum_{k=0}^{t-1}(1-\gamma)^{k+1} \boldsymbol{\delta}(t-k-1) .
\end{aligned}
$$

From (1) and (4), we have

$$
\begin{gathered}
\Delta \mathbf{x}_{i}(t+1)=\mathbf{A}(t) \Delta \mathbf{x}_{i}(t)+\mathbf{B}\left(\Delta \mathbf{u}_{i}(t)-\mathbf{w}_{i}(t)\right) \\
\mathbf{e}_{i}(t)=\mathbf{C} \Delta \mathbf{x}_{i}(t)-\mathbf{v}_{i}(t) .
\end{gathered}
$$


Taking expectation on both sides of (12) and expanding expression from $\mathscr{E}\left[\Delta \mathbf{x}_{i}(t+1)\right]$ to $\mathscr{E}\left[\Delta \mathbf{x}_{i}(1)\right]$, we obtain

$$
\begin{aligned}
\mathscr{E}\left[\Delta \mathbf{x}_{i}(t+1)\right]= & \mathbf{A}(t) \mathscr{E}\left[\Delta \mathbf{x}_{i}(t)\right]+\mathbf{B} \mathscr{E}\left[\Delta \mathbf{u}_{i}(t)\right], \\
\mathscr{E}\left[\Delta \mathbf{x}_{i}(t)\right]= & \mathbf{A}(t-1) \mathscr{E}\left[\Delta \mathbf{x}_{i}(t-1)\right] \\
& +\mathbf{B} \mathscr{E}\left[\Delta \mathbf{u}_{i}(t-1)\right], \\
& \vdots \\
\mathscr{E}\left[\Delta \mathbf{x}_{i}(1)\right]= & \mathbf{A}(0) \mathscr{E}\left[\Delta \mathbf{x}_{i}(0)\right]+\mathbf{B} \mathscr{E}\left[\Delta \mathbf{u}_{i}(0)\right] .
\end{aligned}
$$

The above expression can be arranged later (this paper prescribes $\prod_{\tau=\tau_{1}}^{\tau_{2}}=\mathbf{I}$ when $\tau_{2}<\tau_{1}$ )

$$
\begin{aligned}
\mathscr{E}\left[\Delta \mathbf{x}_{i}(t+1)\right] & =\sum_{\tau=0}^{t} \prod_{\nu=\tau+1}^{t} \mathbf{A}(\nu) \mathbf{B} \mathscr{E}\left[\Delta \mathbf{u}_{i}(\tau)\right] \\
\mathscr{E}\left[\mathscr{A}\left[\mathbf{e}_{i}(t+1)\right]\right] & =\mathbf{C} \mathscr{E}\left[\mathscr{A}\left[\Delta \mathbf{x}_{i}(t+1)\right]\right] \\
& =\mathbf{C} \sum_{\tau=0}^{t} \prod_{\nu=\tau+1}^{t} \mathbf{A}(\nu) \mathbf{B} \mathscr{E}\left[\mathscr{A}\left[\Delta \mathbf{u}_{i}(\tau)\right]\right] .
\end{aligned}
$$

For any $a>1$ and any $\lambda>1$, denote

$$
\begin{gathered}
\left\|\mathscr{E}\left[\mathscr{A}\left[\Delta \mathbf{u}_{i}\right]\right]\right\|_{(\lambda, a)} \triangleq \max _{t \in\{0,1, \ldots, T\}} a^{-\lambda t}\left\|\mathscr{E}\left[\mathscr{A}\left[\Delta \mathbf{u}_{i}(t)\right]\right]\right\|_{2}, \\
\left\|\mathscr{E}\left[\mathscr{A}\left[\mathbf{e}_{i}\right]\right]\right\|_{(\lambda, a)} \triangleq \max _{t \in\{0,1, \ldots, T\}} a^{-\lambda t}\left\|\mathscr{E}\left[\mathscr{A}\left[\mathbf{e}_{i}(t)\right]\right]\right\|_{2} .
\end{gathered}
$$

Lemma 2. For all $a>1$, for all $\lambda>1$, and for all $i \in \mathbb{Z}_{+}$,

$$
\begin{gathered}
\gamma \max _{t \in\{0, \ldots, T\}} a^{-\lambda t} \sum_{k=0}^{t}(1-\gamma)^{k} \sum_{\tau=0}^{t-k-1} a^{t-k-\tau}\left\|\mathscr{E}\left[\mathscr{A}\left[\Delta \mathbf{u}_{i}(\tau)\right]\right]\right\|_{2} \\
\leq \frac{\left\|\mathscr{E}\left[\mathscr{A}\left[\Delta \mathbf{u}_{i}\right]\right]\right\|_{(\lambda, a)}}{a^{\lambda-1}-1} .
\end{gathered}
$$

Proof. From (17), we have

$$
\begin{aligned}
& \gamma \max _{t \in\{0, \ldots, T\}} a^{-\lambda t} \sum_{k=0}^{t}(1-\gamma)^{k} \\
& \quad \times \sum_{\tau=0}^{t-k-1} a^{t-k-\tau}\left\|\mathscr{E}\left[\mathscr{A}\left[\Delta \mathbf{u}_{i}(\tau)\right]\right]\right\|_{2} \\
& \leq \gamma \max _{t \in\{0, \ldots, T\}} \sum_{k=0}^{t}(1-\gamma)^{k} \\
& \times \sum_{\tau=0}^{t-k-1} a^{-\lambda \tau}\left\|\mathscr{E}\left[\mathscr{A}\left[\Delta \mathbf{u}_{i}(\tau)\right]\right]\right\|_{2} a^{-(\lambda-1) t} a^{(\lambda-1) \tau}
\end{aligned}
$$

$$
\begin{aligned}
\leq & \gamma_{t \in\{0, \ldots, T\}} \sum_{k=0}^{t}(1-\gamma)^{k}\left\|\mathscr{E}\left[\mathscr{A}\left[\Delta \mathbf{u}_{i}\right]\right]\right\|_{(\lambda, a)} \\
& \times \sum_{\tau=0}^{t-k-1} a^{-(\lambda-1) t} a^{(\lambda-1) \tau} \\
\leq & \left\|\mathscr{E}\left[\mathscr{A}\left[\Delta \mathbf{u}_{i}\right]\right]\right\|_{(\lambda, a)} \max _{t \in\{0, \ldots, T\}} \gamma \\
& \times \sum_{k=0}^{t}(1-\gamma)^{k} \frac{a^{-(\lambda-1) k}-a^{-(\lambda-1) t}}{a^{\lambda-1}-1} \\
\leq & \frac{\left\|\mathscr{E}\left[\mathscr{A}\left[\Delta \mathbf{u}_{i}\right]\right]\right\|_{(\lambda, a)}}{a^{\lambda-1}-1} \max _{t \in\{0, \ldots, T\}} \gamma \frac{1-(1-\gamma)^{t+1}}{1-(1-\gamma)} \\
\leq & \frac{\left\|\mathscr{E}\left[\mathscr{A}\left[\Delta \mathbf{u}_{i}\right]\right]\right\|_{(\lambda, a)}}{a^{\lambda-1}-1} .
\end{aligned}
$$

Theorem 3. For the system with network communication described in Section 2 and the iterative learning controller (5), suppose

$$
\rho \triangleq\|\mathbf{I}-\gamma \mathbf{L} \mathbf{C B}\|<1
$$

Then for all $\mathbf{A}_{0}, \ldots, \mathbf{A}_{T} \in \mathbb{R}^{n \times n}$, for all $\epsilon>0$, there exist $a>1$ and $\lambda>1$ such that

$$
\lim _{i \rightarrow \infty}\left\|\mathscr{E}\left[\mathbf{e}_{i}\right]\right\|_{(\lambda, a)}<\epsilon .
$$

Proof. From definition of average operator, note the relation

$$
\begin{aligned}
\mathscr{A}\left[\Delta \mathbf{u}_{i+1}(t)\right] & =\frac{1}{i+1}\left(\Delta \mathbf{u}_{i+1}(t)+\sum_{j=1}^{i} \Delta \mathbf{u}_{j}(t)\right) \\
& =\frac{1}{i+1}\left(\Delta \mathbf{u}_{i+1}(t)+i \mathscr{A}\left[\Delta \mathbf{u}_{i}(t)\right]\right) .
\end{aligned}
$$

Applying the ensemble operator $\mathscr{E}[\cdot]$ to both sides of (21) and substituting the relationship (8), we can obtain

$$
\begin{aligned}
\mathscr{E}[\mathscr{A}[ & \left.\left.\Delta \mathbf{u}_{i+1}(t)\right]\right] \\
= & \frac{1}{i+1} \mathscr{E}\left[\Delta \mathbf{u}_{i+1}(t)\right]+\frac{i}{i+1} \mathscr{E}\left[\mathscr{A}\left[\Delta \mathbf{u}_{i}(t)\right]\right] \\
= & \frac{\gamma}{i+1}\left(\mathscr{E}\left[\mathscr{A}\left[\Delta \mathbf{u}_{i}(t)\right]\right]-(i+1) \mathbf{L} \mathscr{E}\left[\mathscr{A}\left[\mathbf{e}_{i}(t+1)\right]\right]\right) \\
& +\frac{1-\gamma}{i+1}\left(\mathscr{E}\left[\Delta \mathbf{u}_{i+1}(t-1)\right]+\boldsymbol{\delta}(t)\right) \\
& +\frac{i}{i+1} \mathscr{E}\left[\mathscr{A}\left[\Delta \mathbf{u}_{i}(t)\right]\right] .
\end{aligned}
$$


Substituting (11) into (22) leads to the following relationship:

$$
\begin{aligned}
\mathscr{E}[\mathscr{A}[ & \left.\left.\Delta \mathbf{u}_{i+1}(t)\right]\right] \\
= & \frac{i+\gamma}{i+1} \mathscr{E}\left[\mathscr{A}\left[\Delta \mathbf{u}_{i}(t)\right]\right]-\gamma \mathbf{L} \mathscr{E}\left[\mathscr{A}\left[\mathbf{e}_{i}(t+1)\right]\right] \\
& +\frac{\gamma}{i+1} \sum_{k=0}^{t-1}(1-\gamma)^{k+1} \mathscr{E}\left[\mathscr{A}\left[\Delta \mathbf{u}_{i}(t-k-1)\right]\right] \\
& -\gamma \mathbf{L} \sum_{k=0}^{t-1}(1-\gamma)^{k+1} \mathscr{E}\left[\mathscr{A}\left[\mathbf{e}_{i}(t-k)\right]\right] \\
& +\frac{1-\gamma}{i+1}\left(\sum_{k=0}^{t-1}(1-\gamma)^{k+1} \boldsymbol{\delta}(t-k-1)+\boldsymbol{\delta}(t)\right) \\
= & \frac{i+\gamma}{i+1} \mathscr{E}\left[\mathscr{A}\left[\Delta \mathbf{u}_{i}(t)\right]\right]+\frac{\gamma}{i+1} \\
& \times \sum_{k=0}^{t-1}(1-\gamma)^{k+1} \mathscr{E}\left[\mathscr{A}\left[\Delta \mathbf{u}_{i}(t-k-1)\right]\right] \\
& -\gamma \mathbf{L} \sum_{k=0}^{t}(1-\gamma)^{k} \mathscr{E}\left[\mathscr{A}\left[\mathbf{e}_{i}(t-k+1)\right]\right] \\
& +\frac{1}{i+1} \sum_{k=0}^{t}(1-\gamma)^{k+1} \boldsymbol{\delta}(t-k) .
\end{aligned}
$$

Now let us handle the third term on the right hand side of (23); we will express $\mathscr{E}\left[\mathscr{A}\left[\mathbf{e}_{i}(t-k+1)\right]\right]$ with $\mathscr{E}\left[\mathscr{A}\left[\mathbf{u}_{i}\right]\right]$. Substituting the state error dynamics (14) into (23) leads to the following relationship:

$$
\begin{aligned}
\mathscr{E}[\mathscr{A}[ & \left.\left.\Delta \mathbf{u}_{i+1}(t)\right]\right] \\
= & \frac{i+\gamma}{i+1} \mathscr{E}\left[\mathscr{A}\left[\Delta \mathbf{u}_{i}(t)\right]\right]+\frac{\gamma}{i+1} \\
& \times \sum_{k=0}^{t-1}(1-\gamma)^{k+1} \mathscr{E}\left[\mathscr{A}\left[\Delta \mathbf{u}_{i}(t-k-1)\right]\right] \\
& -\gamma \mathbf{L C} \sum_{k=0}^{t}(1-\gamma)^{k} \\
& \times \sum_{\tau=0}^{t-k} \prod_{\nu=\tau+1}^{t-k} \mathbf{A}(\nu) \mathbf{B} \mathscr{E}\left[\mathscr{A}\left[\Delta \mathbf{u}_{i}(\tau)\right]\right]+\boldsymbol{\mu}_{i}(t) \\
= & \frac{i+\gamma}{i+1} \mathscr{E}\left[\mathscr{A}\left[\Delta \mathbf{u}_{i}(t)\right]\right]+\frac{\gamma}{i+1} \\
& \times \sum_{k=1}^{t}(1-\gamma)^{k} \mathscr{E}\left[\mathscr{A}\left[\Delta \mathbf{u}_{i}(t-k)\right]\right] \\
& -\gamma \mathbf{L C} \sum_{k=0}^{t}(1-\gamma)^{k}
\end{aligned}
$$

$$
\begin{aligned}
& \times \sum_{\tau=0}^{t-k-1} \prod_{\nu=\tau+1}^{t-k} \mathbf{A}(\nu) \mathbf{B} \mathscr{E}\left[\mathscr{A}\left[\Delta \mathbf{u}_{i}(\tau)\right]\right] \\
& -\gamma \mathbf{L C} \sum_{k=0}^{t}(1-\gamma)^{k} \mathbf{B} \mathscr{E}\left[\mathscr{A}\left[\Delta \mathbf{u}_{i}(t-k)\right]\right]+\boldsymbol{\mu}_{i}(t),
\end{aligned}
$$

where $\boldsymbol{\mu}_{i}(t) \triangleq(1 /(i+1)) \sum_{k=0}^{t}(1-\gamma)^{k+1} \boldsymbol{\delta}(t-k)$.

Next, combining analogous terms on the right hand of (22), we obtain

$$
\begin{aligned}
\mathscr{E}[\mathscr{A}[ & \left.\left.\Delta \mathbf{u}_{i+1}(t)\right]\right] \\
= & \frac{i+\gamma}{i+1} \mathscr{E}\left[\mathscr{A}\left[\Delta \mathbf{u}_{i}(t)\right]\right]+\frac{\gamma}{i+1} \\
& \times \sum_{k=1}^{t}(1-\gamma)^{k} \mathscr{E}\left[\mathscr{A}\left[\Delta \mathbf{u}_{i}(t-k)\right]\right] \\
& -\gamma \mathbf{L C} \sum_{k=0}^{t}(1-\gamma)^{k} \\
& \times \sum_{\tau=0}^{t-k-1} \prod_{\nu=\tau+1}^{t-k} \mathbf{A}(\nu) \mathbf{B} \mathscr{E}\left[\mathscr{A}\left[\Delta \mathbf{u}_{i}(\tau)\right]\right] \\
& -\gamma \mathbf{L C} \sum_{k=1}^{t}(1-\gamma)^{k} \mathbf{B} \mathscr{E}\left[\mathscr{A}\left[\Delta \mathbf{u}_{i}(t-k)\right]\right] \\
& +\gamma \mathbf{L C} \sum_{k=0}^{t}(1-\gamma)^{k} \\
& -\gamma \mathbf{L C B} \mathscr{E}\left[\mathscr{A}\left[\Delta \mathbf{u}_{i}(t)\right]\right]+\boldsymbol{\mu}_{i}(t) \\
= & \left.\frac{i+\gamma}{i+1} \mathbf{I}-\gamma \mathbf{L C B}\right) \mathscr{E}\left[\mathscr{A}\left[\Delta \mathbf{u}_{i}(t)\right]\right] \\
& +\left(\frac{\gamma}{i+1} \mathbf{I}-\gamma \mathbf{L C B}\right) \sum_{k=1}^{t}(1-\gamma)^{k} \mathscr{E}\left[\mathscr{A}\left[\Delta \mathbf{u}_{i}(t-k)\right]\right]
\end{aligned}
$$

The relationship (25) can be rewritten as follows:

$$
\begin{aligned}
\mathscr{E}\left[\mathscr{A}\left[\Delta \mathbf{u}_{i+1}(t)\right]\right] \\
=\left(\frac{i+\gamma}{i+1} \mathbf{I}-\gamma \mathbf{L C B}\right) \mathscr{E}\left[\mathscr{A}\left[\Delta \mathbf{u}_{i}(t)\right]\right] \\
\quad+\left(\frac{\gamma}{i+1} \mathbf{I}-\gamma \mathbf{L C B}\right) \sum_{\tau=0}^{t-1}(1-\gamma)^{t-\tau} \mathscr{E}\left[\mathscr{A}\left[\Delta \mathbf{u}_{i}(\tau)\right]\right]
\end{aligned}
$$




$$
\begin{aligned}
& -\gamma \mathbf{L C} \sum_{k=0}^{t}(1-\gamma)^{k} \\
& \times \sum_{\tau=0}^{t-k-1} \prod_{\nu=\tau+1}^{t-k} \mathbf{A}(\nu) \mathbf{B} \mathscr{E}\left[\mathscr{A}\left[\Delta \mathbf{u}_{i}(\tau)\right]\right]+\boldsymbol{\mu}_{i}(t) .
\end{aligned}
$$

To simplify expression of $((i+\gamma) /(i+1)) \mathbf{I}-\gamma \mathbf{L C B},(\gamma /(i+1)) \mathbf{I}-$ $\gamma \mathbf{L C B}$, and $\gamma \mathbf{L C} \sum_{k=0}^{t}(1-\gamma)^{k}$, we choose $a>1$ and $\lambda>1$ such that

$$
\begin{gathered}
a>\|\mathbf{A}(\nu)\| \quad \forall \nu \in\{0, \ldots, T\}, \\
\frac{\gamma\|\mathbf{L C} \mathbf{B}\|+\gamma+\|\mathbf{L C}\|\|\mathbf{B}\|}{a^{\lambda-1}-1}<\frac{1-\rho}{4}, \\
\|\mathbf{C}\|\|\mathbf{B}\| \frac{1}{a^{\lambda-1}-1} \frac{2 \alpha}{1-\rho}<\epsilon,
\end{gathered}
$$

where

$$
\begin{aligned}
\alpha & \triangleq \max _{t \in\{0, \ldots, T\}} \frac{a^{-\lambda t}}{2} \sum_{k=0}^{t}(1-\gamma)^{k+1}\|\boldsymbol{\delta}(t-k)\|_{2} \\
& \geq \max _{t \in\{0, \ldots, T\}} a^{-\lambda t}\left\|\boldsymbol{\mu}_{i}(t)\right\|_{2} .
\end{aligned}
$$

Taking $\lambda$-norm on both sides of (26), we obtain

$$
\begin{aligned}
\| \mathscr{E}[\mathscr{A}[ & \left.\left.\Delta \mathbf{u}_{i+1}\right]\right] \|_{(\lambda, a)} \\
\leq & \left\|\mathbf{I}-\gamma \mathbf{L} \mathbf{C B}-\frac{1-\gamma}{i+1} \mathbf{I}\right\|\left\|\mathscr{E}\left[\mathscr{A}\left[\Delta \mathbf{u}_{i}\right]\right]\right\|_{(\lambda, a)} \\
& +\max _{t \in\{0, \ldots, T\}} a^{-\lambda t}\left\|\boldsymbol{\mu}_{i}(t)\right\|_{2} \\
& +\left\|\frac{\gamma}{i+1} \mathbf{I}-\gamma \mathbf{L C B}\right\|_{t \in\{0, \ldots, T\}} \max ^{-\lambda t} \\
& \times \sum_{\tau=0}^{t-1}(1-\gamma)^{t-\tau}\left\|\mathscr{E}\left[\mathscr{A}\left[\Delta \mathbf{u}_{i}(\tau)\right]\right]\right\|_{2} \\
& +\gamma\|\mathbf{L C}\|\|\mathbf{B}\| \max _{t \in\{0, \ldots, T\}} a^{-\lambda t} \\
& \times \sum_{k=0}^{t}(1-\gamma)^{k} \sum_{\tau=0}^{t-k-1} a^{t-k-\tau}\left\|\mathscr{E}\left[\mathscr{A}\left[\Delta \mathbf{u}_{i}(\tau)\right]\right]\right\|_{2} .
\end{aligned}
$$

Using Lemma 2, it can be proved that, for all, $a>1$, for all $\lambda>1$, and for all $i \in \mathbb{Z}_{+}$,

$$
\begin{gathered}
\max _{t \in\{0, \ldots, T\}} a^{-\lambda t} \sum_{\tau=0}^{t-1}(1-\gamma)^{t-\tau}\left\|\mathscr{E}\left[\mathscr{A}\left[\Delta \mathbf{u}_{i}(\tau)\right]\right]\right\|_{2} \\
\leq \frac{\left\|\mathscr{E}\left[\mathscr{A}\left[\Delta \mathbf{u}_{i}\right]\right]\right\|_{(\lambda, a)}}{a^{\lambda-1}-1} .
\end{gathered}
$$

Combining Lemma 2, (31) and (32) yields

$$
\begin{aligned}
\left\|\mathscr{E}\left[\mathscr{A}\left[\Delta \mathbf{u}_{i+1}\right]\right]\right\|_{(\lambda, a)} \\
\leq\left(\rho+\frac{1-\gamma}{i+1}+\frac{\gamma\|\mathbf{L C B}\|+\gamma+\|\mathbf{L C}\|\|\mathbf{B}\|}{a^{\lambda-1}-1}\right) \\
\quad \times\left\|\mathscr{E}\left[\mathscr{A}\left[\Delta \mathbf{u}_{i}(t)\right]\right]\right\|_{(\lambda, a)}+\alpha .
\end{aligned}
$$

There exists $M \in \mathbb{Z}_{+}$such that $(1-\gamma) /(i+1)<(1-\rho) / 4$ when $i \geq M$. Now for $i>M$, (28) and (33) imply that

$$
\begin{aligned}
\left\|\mathscr{E}\left[\mathscr{A}\left[\Delta \mathbf{u}_{i+1}\right]\right]\right\|_{(\lambda, a)} \leq \frac{1+\rho}{2}\left\|\mathscr{E}\left[\mathscr{A}\left[\Delta \mathbf{u}_{i}\right]\right]\right\|_{(\lambda, a)}+\alpha \\
\left\|\mathscr{E}\left[\mathscr{A}\left[\Delta \mathbf{u}_{i+1}\right]\right]\right\|_{(\lambda, a)} \\
\leq\left(\frac{1+\rho}{2}\right)^{i+1-M}\left\|\mathscr{E}\left[\mathscr{A}\left[\Delta \mathbf{u}_{M}\right]\right]\right\|_{(\lambda, a)} \\
\quad+\sum_{j=0}^{i-M}\left(\frac{1+\rho}{2}\right)^{j} \alpha \\
\leq\left(\frac{1+\rho}{2}\right)^{i+1-M}\left\|\mathscr{E}\left[\mathscr{A}\left[\Delta \mathbf{u}_{M}\right]\right]\right\|_{(\lambda, a)} \\
+\frac{1-((1+\rho) / 2)^{i+1-M}}{1-((1+\rho) / 2)} \alpha .
\end{aligned}
$$

Consequently, we obtain

$$
\lim _{i \rightarrow \infty}\left\|\mathscr{E}\left[\mathscr{A}\left[\Delta \mathbf{u}_{i+1}\right]\right]\right\|_{(\lambda, a)} \leq \frac{2 \alpha}{1-\rho} .
$$

According to the relationship (14) between the input error and output error, we have

$$
\mathscr{E}\left[\mathscr{A}\left[\mathbf{e}_{i}(t)\right]\right]=\mathbf{C} \sum_{\tau=0}^{t-1} \prod_{\nu=\tau+1}^{t-1} \mathbf{A}(\nu) \mathbf{B} \mathscr{E}\left[\mathscr{A}\left[\Delta \mathbf{u}_{i}(\tau)\right]\right] .
$$

Similar to the proof of Lemma 2, one can prove that

$$
\begin{gathered}
\max _{t \in\{0, \ldots, T\}} a^{-\lambda t}\left\|\mathscr{E}\left[\mathscr{A}\left[\mathbf{e}_{i}(t)\right]\right]\right\|_{2} \\
\leq\|\mathbf{C}\|\|\mathbf{B}\| \max _{t \in\{0, \ldots, T\}} a^{-\lambda t} \sum_{\tau=0}^{t-1} a^{t-1-\tau}\left\|\mathscr{E}\left[\mathscr{A}\left[\Delta \mathbf{u}_{i}\right]\right]\right\|_{2} \\
\leq\|\mathbf{C}\|\|\mathbf{B}\| \frac{\left\|\mathscr{E}\left[\mathscr{A}\left[\Delta \mathbf{u}_{i}\right]\right]\right\|_{(\lambda, a)}}{a^{\lambda-1}-1} .
\end{gathered}
$$

Finally, from (29), (35), and (37), we can obtain

$$
\lim _{i \rightarrow \infty}\left\|\mathscr{E}\left[\mathscr{A}\left[\mathbf{e}_{i+1}\right]\right]\right\|_{(\lambda, a)} \leq\|\mathbf{C}\|\|\mathbf{B}\| \frac{2 \alpha}{1-\rho} \frac{1}{a^{\lambda-1}-1}<\epsilon
$$

because lambda can be chosen arbitrarily large in (38).

This completes the proof. 


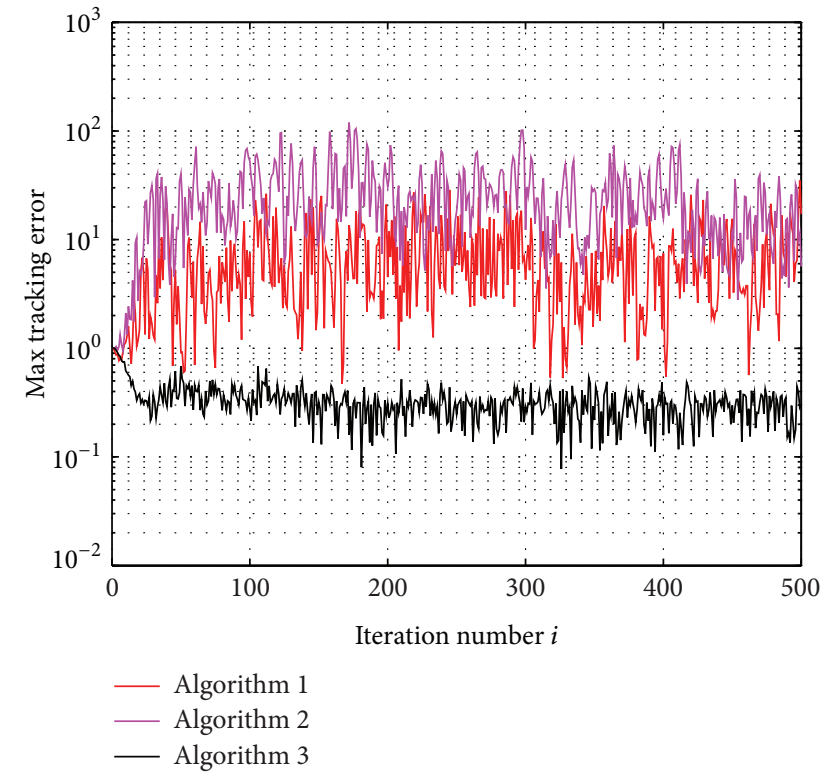

FIGURE 2: The max tracking error versus iteration with $5 \%$ packet dropout.

Remark 4. In this paper, we consider D-type iterative learning control with average operator, and the result obtained can be extended to P-type iterative learning control with average operator.

\section{Numerical Examples}

In this simulation test, let us consider system (1) and matrices given by

$$
\begin{gathered}
\mathbf{A}(t)=\left[\begin{array}{cc}
0 & 1 \\
-1-10^{-3} t & -2-10^{-3} t
\end{array}\right], \\
\mathbf{B}=\left[\begin{array}{l}
0 \\
1
\end{array}\right], \quad \mathbf{C}=\left[\begin{array}{ll}
1 & 1
\end{array}\right] .
\end{gathered}
$$

The random noises $\mathbf{w}_{i}(t)$ and $\mathbf{v}_{i}(t)$ have uniform distribution on the intervals $[-0.05,0.05]$ and $[-0.01,0.01]$, respectively. In this control problem, the desired output trajectory for $t \in\{0,1, \ldots, 50\}$ is given to be $\mathbf{y}_{d}(t)=\sin (2 \pi t / 50)$, and the initial states $x_{1 i}(0)$ and $x_{2 i}(0)$ have uniform distribution on the intervals $[-0.01,0.01]$ and $[-0.02,0.02]$, respectively. The fixed time interval $T$ is 50 . The control profile of the first iteration is $u_{1}(t)=0$. Random packet dropout in controlleractuator channel is subject to Bernoulli distribution of expected value $\gamma$ ( 1 means transmission success while 0 means transmission failure).

For expected value $\gamma=0.95$, we compare our algorithm with the other 2 algorithms.

Algorithm 1 (classic ILC). The control signal is constructed as

$$
\begin{aligned}
u_{i+1}(t)= & \gamma_{i+1}(t)\left(u_{i}(t)+\mathbf{L} e_{i}(t+1)\right) \\
& +\left(1-\gamma_{i+1}(t)\right) u_{i+1}(t-1)
\end{aligned}
$$

with $\mathbf{L}=0.2$ satisfying $\|1-\gamma \mathbf{L C B}\|=0.81<1$.

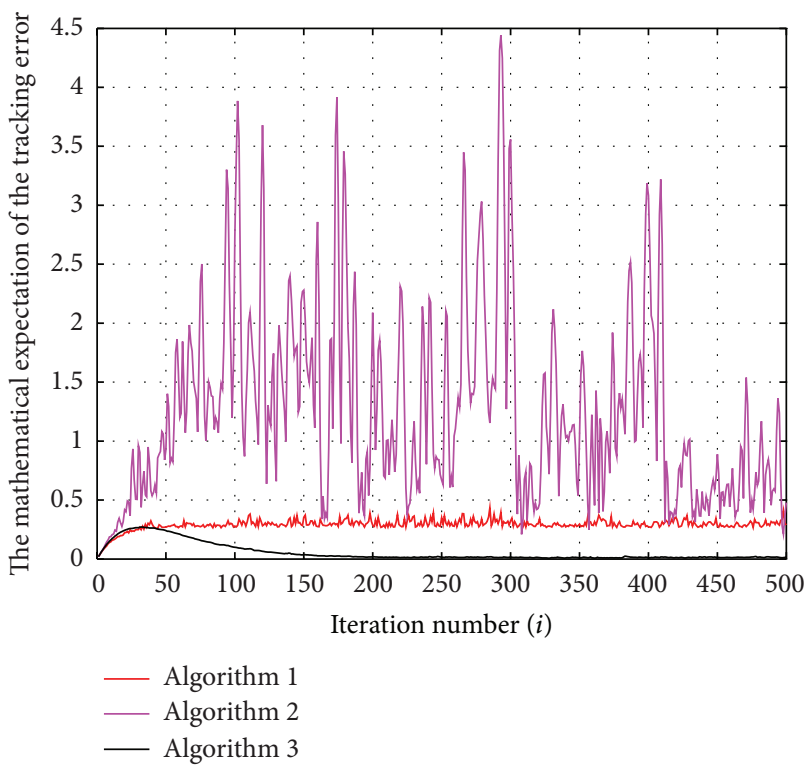

FIGURE 3: The mathematical expectation of the tracking error versus iteration with $5 \%$ packet dropout.

Algorithm 2 (zero-input scheme with average ILC). The control signal is constructed as

$$
u_{i+1}(t)=\gamma_{i+1}(t)\left(\mathscr{A}\left[u_{i}(t)\right]+(i+1) L \mathscr{A}\left[e_{i}(t+1)\right]\right)
$$

with $L=0.2$.

Algorithm 3. Now, we consider the proposed algorithm. From (2) and (5), the control signal $u_{i+1}(t)$ is constructed as

$$
\begin{aligned}
u_{i+1}(t)= & \gamma_{i+1}(t) \hat{u}_{i+1}(t)+\left(1-\gamma_{i+1}(t)\right) u_{i+1}(t-1) \\
= & \gamma_{i+1}(t)\left(\mathscr{A}\left[u_{i}(t)\right]+(i+1) \mathbf{L} \mathscr{A}\left[e_{i}(t+1)\right]\right) \\
& +\left(1-\gamma_{i+1}(t)\right) u_{i+1}(t-1),
\end{aligned}
$$

where the learning gain $L=0.2$ and expected value $\gamma=0.95$.

As shown in Figure 2, the tracking error profiles for the proposed algorithm are much lower than the other two algorithms with $5 \%$ packet dropout. In Figure 3, the mathematical expectation of the tracking error versus iterations is shown, and the proposed hold-input scheme with average ILC achieves the convergent performance.

\section{Conclusion}

In this work we address a remote control system problem with random packet dropout in controller-actuator channel. The hold-input scheme with average ILC is applied to handle this remote control problem with repeated tracking tasks. Through analysis we illustrate the desired convergence property of the hold-input scheme with average ILC. In our future work, we will also explore the extension to more generic stochastic process such as Markov chain. 


\section{Nomenclature}

$\mathbb{R}: \quad$ The set of all real numbers

$\mathbb{Z}_{+}$: The set of all positive integers

$\mathscr{A}\left[\mathbf{v}_{m}\right]$ : The average operator $\mathscr{A}\left[\mathbf{v}_{m}\right]=(1 / m) \sum_{i=1}^{m} \mathbf{v}_{i}$

$\mathscr{E}[\cdot]$ : The expected value of a random variable

$\mathscr{P}[\cdot]: \quad$ The probability of an event

$\|\cdot\|: \quad$ The maximal singular value of a matrix

$\|\cdot\|_{2}$ : The Euclidean norm of a vector

I: Identity matrix of appropriate dimensions

0: $\quad$ Zero matrix of appropriate dimensions.

\section{Acknowledgment}

This work is supported by the 973 program of China (Grant no. 2009CB320603).

\section{References}

[1] S. Arimoto, S. Kawamura, and F. Miyazaki, "Berrering operation of robots by learning," Journal of Robot Systems, vol. 1, no. 1, pp. 123-140, 1984.

[2] Z. Bien and K. M. Huh, "Higher-order iterative learning control algorithm," IEE Proceedings D, vol. 136, no. 3, pp. 105-112, 1989.

[3] Y. Chen and K. L. Moore, "Harnessing the nonrepetitiveness in iterative learning control," in Proceedings of the 41st IEEE Conference on Decision and Control, pp. 3350-3355, Las Vegas, Nev, USA, December 2002.

[4] K. L. Moore, Y. Chen, and H.-S. Ahn, "Iterative learning control: A tutorial and big picture view," in Proceedings of the 45th IEEE Conference on Decision and Control (CDC '06), pp. 2352-2357, December 2006.

[5] D. A. Bristow, M. Tharayil, and A. G. Alleyne, "A survey of iterative learning control: A learning-based method for highperformance tracking control," IEEE Control Systems Magazine, vol. 26, no. 3, pp. 96-114, 2006.

[6] Y. Chen, Z. Gong, and C. Wen, "Analysis of a high-order iterative learning control algorithm for uncertain nonlinear systems with state delays," Automatica, vol. 34, no. 3, pp. 345353, 1998.

[7] A. Tayebi and M. B. Zaremba, "Robust iterative learning control design is straightforward for uncertain LTI systems satisfying the robust performance condition," IEEE Transactions on Automatic Control, vol. 48, no. 1, pp. 101-106, 2003.

[8] C. Yin, J.-X. Xu, and Z. Hou, "A high-order internal model based iterative learning control scheme for nonlinear systems with time-iteration-varying parameters," IEEE Transactions on Automatic Control, vol. 55, no. 11, pp. 2665-2670, 2010.

[9] S. S. Saab, "A discrete-time stochastic learning control algorithm," IEEE Transactions on Automatic Control, vol. 46, no. 6, pp. 877-887, 2001.

[10] M. Sun and D. Wang, "Iterative learning control with initial rectifying action," Automatica, vol. 38, no. 8, pp. 1177-1182, 2002.

[11] Y. Chen, C. Wen, Z. Gong, and M. Sun, "An iterative learning controller with initial state learning," IEEE Transactions on Automatic Control, vol. 44, no. 2, pp. 371-376, 1999.

[12] R. Chi, Z. Hou, and J. Xu, "Adaptive ILC for a class of discretetime systems with iteration-varying trajectory and random initial condition," Automatica, vol. 44, no. 8, pp. 2207-2213, 2008.
[13] J.-X. Xu and Y. Tan, Linear and Nonlinear Iterative Learning Control, vol. 291 of Lecture Notes in Control and Information Sciences, Springer, Berlin, Germany, 2003.

[14] H.-S. Ahn, K. L. Moore, and Y. Chen, Iterative Learning Control: Robustness and Monotonic Convergence for Interval Systems, Communications and Control Engineering Series, Springer, London, UK, 2007.

[15] L.-S. Hu, T. Bai, P. Shi, and Z. Wu, "Sampled-data control of networked linear control systems," Automatica, vol. 43, no. 5, pp. 903-911, 2007.

[16] M. B. G. Cloosterman, N. van de Wouw, W. P. M. H. Heemels, and $\mathrm{H}$. Nijmeijer, "Stability of networked control systems with uncertain time-varying delays," IEEE Transactions on Automatic Control, vol. 54, no. 7, pp. 1575-1580, 2009.

[17] J. Nilsson, Real time control systems with delay [Ph.D. thesis], Lund Institute of Technology, 1998.

[18] L. A. Montestruque and P. Antsaklis, "Stability of model-based networked control systems with time-varying transmission times," IEEE Transactions on Automatic Control, vol. 49, no. 9, pp. 1562-1572, 2004.

[19] D. Wu, J. Wu, and S. Chen, "Robust $H_{\infty}$ control for networked control systems with uncertainties and multiple-packet transmission," IET Control Theory \& Applications, vol. 4, no. 5, pp. 701-709, 2010.

[20] L. Bakule and M. De La Sen, "Decentralized stabilization of networked complex composite systems with nonlinear perturbations," in Proceedings of the IEEE International Conference on Control and Automation (ICCA '09), pp. 2272-2277, Christchurch, New Zealand, December 2009.

[21] L. Bakule and M. De La Sen, "Decentralized resilient $H_{\infty}$ observer-based control for a class of uncertain interconnected networked systems," in Proceedings of the American Control Conference (ACC '10), pp. 1338-1343, Baltimore, Md, USA, July 2010.

[22] H.-S. Ahn, K. L. Moore, and Y. Chen, "Discrete-time intermittent iterative learning controller with independent data dropouts," in Proceedings of the 17th World Congress, International Federation of Automatic Control (IFAC'08), Seoul, Korea, July 2008.

[23] H.-S. Ahn, K. L. Moore, and Y. Chen, "Stability of discretetime iterative learning control with random data dropouts and delayed controlled signals in networked control systems," in Proceedings of the 10th International Conference on Control, Automation, Robotics and Vision (ICARCV '08), pp. 757-762, December 2008.

[24] C. Liu, J. Xu, and J. Wu, "Iterative learning control for network systems with communication delay or data dropout," in Proceedings of the 48th IEEE Conference on Decision and Control Held Jointly with the 28th Chinese Control Conference (CDC/ CCC '09), pp. 4858-4863, Shanghai, China, December 2009.

[25] C. Liu, J. Xu, and J. Wu, "Iterative learning control for remote control systems with communication delay and data dropout," Mathematical Problems in Engineering, vol. 2012, Article ID 705474, 14 pages, 2012.

[26] X. Bu, Z. Hou, and F. Yu, "Stability of first and high order iterative learning control with data dropouts," International Journal of Control, Automation and Systems, vol. 9, no. 5, pp. 843-849, 2011.

[27] Y.-J. Pan, H. J. Marquez, T. Chen, and L. Sheng, "Effects of network communications on a class of learning controlled nonlinear systems," International Journal of Systems Science, vol. 40, no. 7, pp. 757-767, 2009. 
[28] X. Bu, F. Yu, Z. Hou, and F. Wang, "Iterative learning control for a class of nonlinear systems with random packet losses," Nonlinear Analysis: Real World Applications, vol. 14, no. 1, pp. 567-580, 2013.

[29] L.-X. Huang and Y. Fang, "Convergence analysis of wireless remote iterative learning control systems with dropout compensation," Mathematical Problems in Engineering, vol. 2013, Article ID 609284, 9 pages, 2013. 


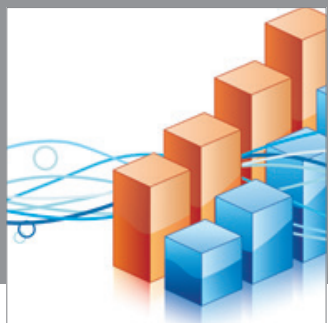

Advances in

Operations Research

mansans

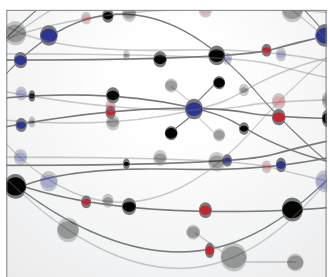

The Scientific World Journal
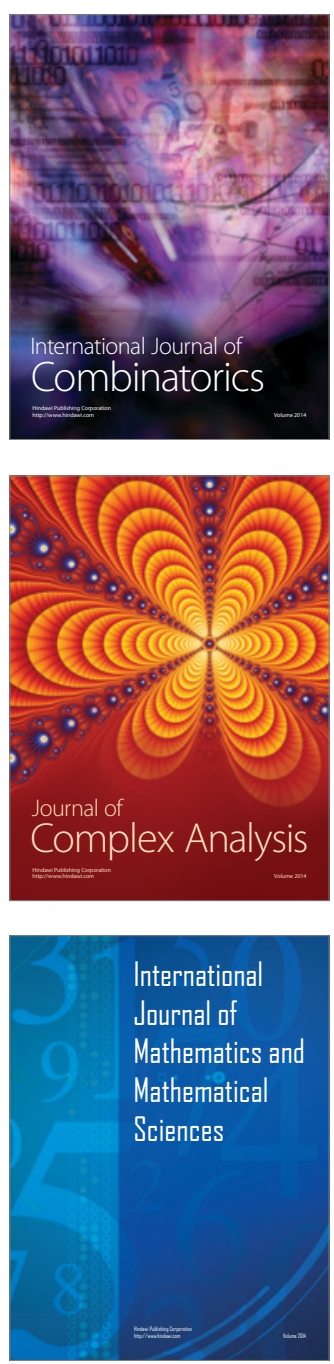
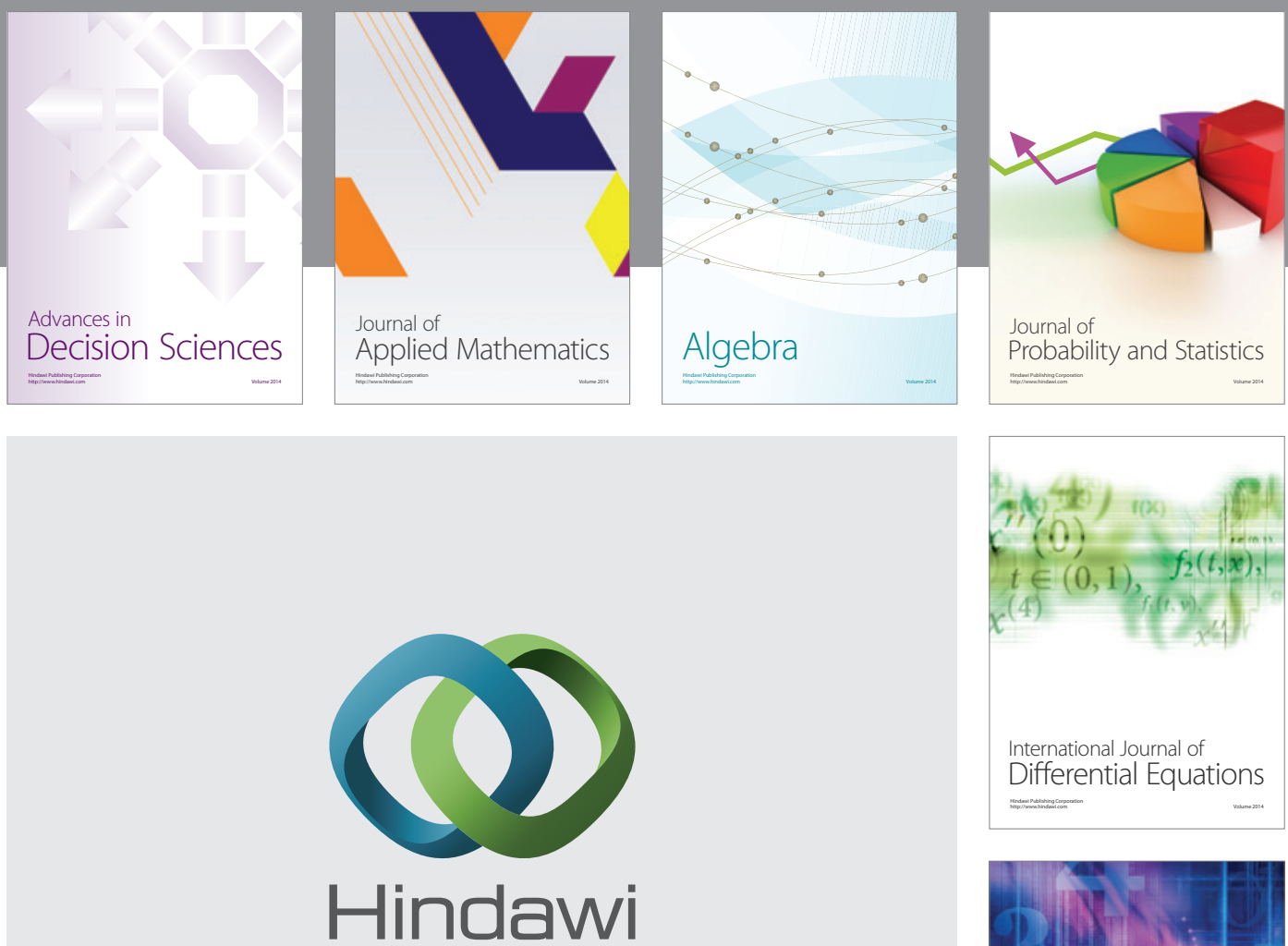

Submit your manuscripts at http://www.hindawi.com
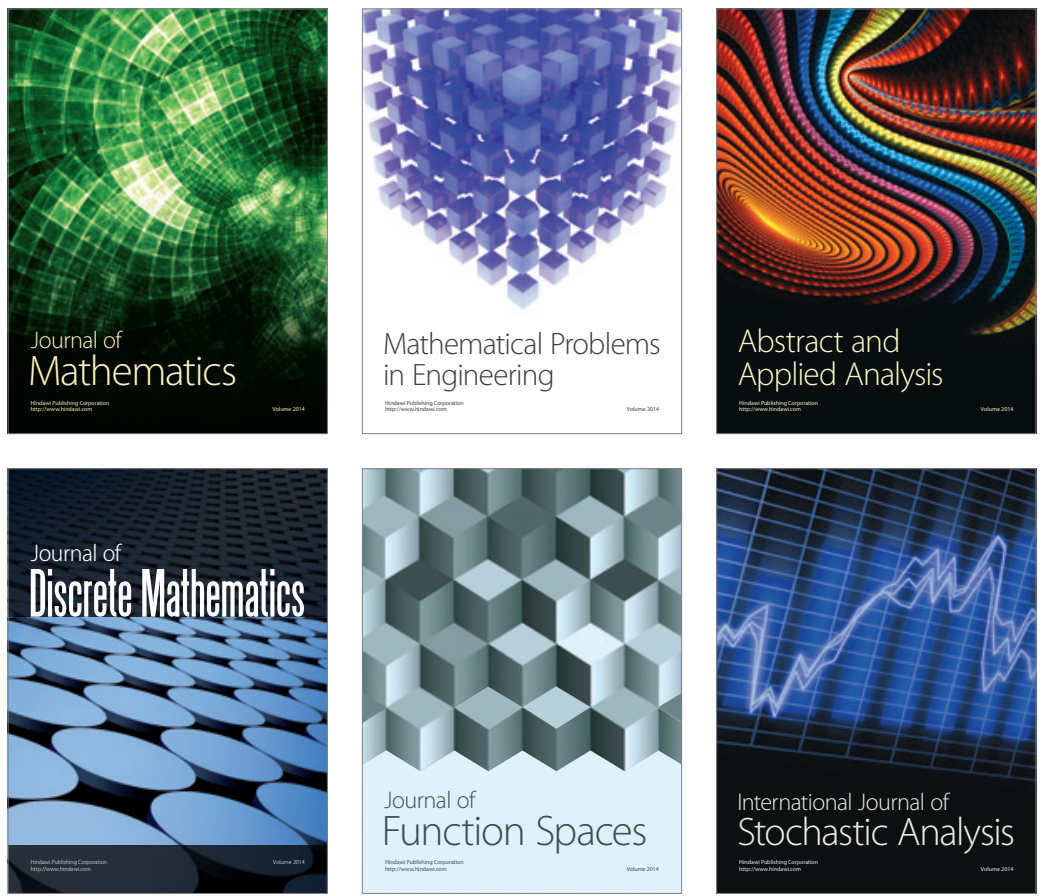

Journal of

Function Spaces

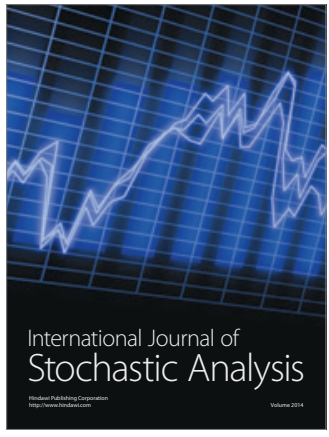

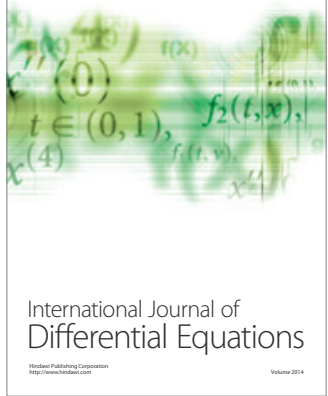
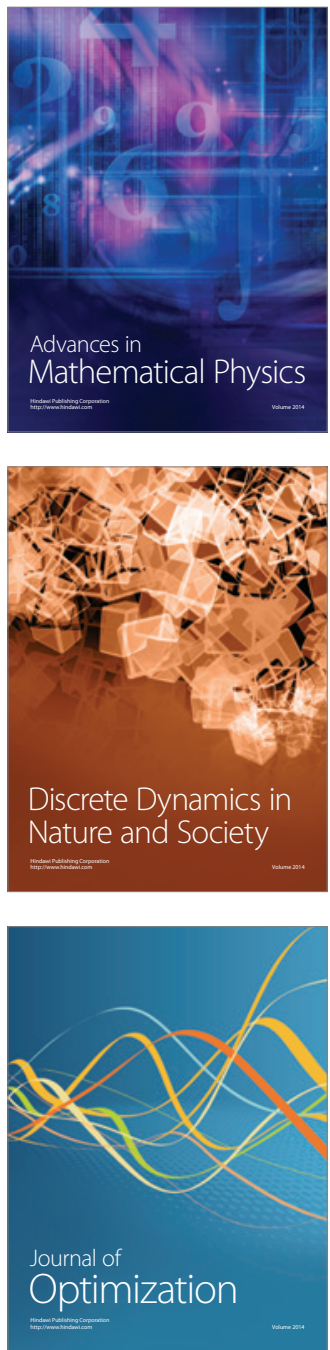